\title{
Fine tuning in general gauge mediation
}

\author{
Tatsuo Kobayashi, ${ }^{a}$ Yuichiro Nakai ${ }^{b}$ and Ryo Takahashi ${ }^{b, c}$ \\ ${ }^{a}$ Department of Physics, Kyoto University, \\ Kyoto 606-8502, Japan \\ ${ }^{b}$ Yukawa Institute for Theoretical Physics, Kyoto University, \\ Kyoto 606-8502, Japan \\ ${ }^{c}$ Max-Planck-Institute für Kernphysik, \\ Postfach 1039 80, 69029 Heidelberg, Germany \\ E-mail: kobayash@gauge.scphys.kyoto-u.ac.jp, \\ ynakai@yukawa.kyoto-u.ac.jp, Ryo.Takahashi@mpi-hd.mpg.de
}

ABSTRACT: We study the fine-tuning problem in the context of general gauge mediation. Numerical analyses toward for relaxing fine-tuning are presented. We analyse the problem in typical three cases of the messenger scale, that is, GUT $\left(2 \times 10^{16} \mathrm{GeV}\right)$, intermediate $\left(10^{10} \mathrm{GeV}\right)$, and relatively low energy $\left(10^{6} \mathrm{GeV}\right)$ scales. In each messenger scale, the parameter space reducing the degree of tuning as around $10 \%$ is found. Certain ratios among gluino mass, wino mass and soft scalar masses are favorable. It is shown that the favorable region becomes narrow as the messenger scale becomes lower, and tachyonic initial conditions of stop masses at the messenger scale are favored to relax the fine-tuning problem for the relatively low energy messenger scale. Our spectra would also be important from the viewpoint of the $\mu-B$ problem.

KEYwords: Supersymmetry Breaking, Beyond Standard Model, Supersymmetric Standard Model

ARXIV EPRINT: 0910.3477 


\section{Contents}

1 Introduction 1

2 Fine tuning in the MSSM 2

3 General gauge mediation 3

4 Numerical analyses $\quad 5$

$\begin{array}{lll}5 & \mu-B \text { problem } & 14\end{array}$

$\begin{array}{lll}6 & \text { Summary } & 16\end{array}$

\section{Introduction}

Low-energy supersymmetric extension of the standard model is one of promising candidates for a new physics at a $\mathrm{TeV}$ scale. The supersymmetry (SUSY) can stabilize the huge hierarchy between the weak scale and the Planck scale. That is a motivation for the lowenergy SUSY. In addition, the three gauge couplings are unified at the grand unified theory (GUT) scale, $2 \times 10^{16} \mathrm{GeV}$, in the minimal supersymmetric standard model (MSSM). Also, supersymmetric standard models have candidates for the dark matter.

Although low-energy SUSY solves the (huge) hierarchy problem between the weak scale and Planck/GUT scale, a few percent of fine-tuning is required in the MSSM as follows. The lightest CP-even Higgs mass $m_{h}$ is predicted as $m_{h} \lesssim M_{Z}$ at the tree level in the MSSM, but that is smaller than the experimental bound $m_{h} \gtrsim 114.4 \mathrm{GeV}$. However, the Higgs mass receives a large radiative correction depending on the averaged stop mass $m_{\tilde{t}}[1-$ 5]. The experimental bound $m_{h} \gtrsim 114.4 \mathrm{GeV}$ requires $m_{\tilde{t}} \gtrsim 1 \mathrm{TeV}$ when $\left|A_{t}\right| / m_{\tilde{t}} \lesssim 1.0$, where $A_{t}$ is the so-called A-term corresponding to the top Yukawa coupling. On the other hand, the stop mass also has a renormalization group (RG) effect on the soft scalar mass $m_{H_{u}}$ of the up-sector Higgs field as [6-12]

$$
\Delta m_{H_{u}}^{2} \sim-\frac{3 y_{t}^{2}}{4 \pi^{2}} m_{\tilde{t}}^{2} \ln \frac{\Lambda}{m_{\tilde{t}}}
$$

where $y_{t}$ is the top Yukawa coupling and $\Lambda$ denotes a cut-off scale of the MSSM such as the Planck scale or GUT scale. This RG effect $\left|\Delta m_{H_{u}}^{2}\right|$ would be comparable to the stop mass with a negative sign. Furthermore, the successful electroweak (EW) symmetry breaking requires

$$
\frac{1}{2} M_{Z}^{2} \sim-\mu^{2}-m_{H_{u}}^{2}
$$


where $\mu$ denotes the supersymmetric mass of the up-sector Higgs field $H_{u}$ and the downsector Higgs field $H_{d}$. If $m_{H_{u}}^{2} \sim-m_{\tilde{t}}^{2}$ and $m_{\tilde{t}}=\mathcal{O}(1) \mathrm{TeV}$, one needs a few percent of fine-tuning between $\mu^{2}$ and $m_{H_{u}}^{2}$ in order to derive the correct value of $M_{Z}$. That is the so-called little hierarchy problem [13-18]. Several works have been done to address this issue [19]-[45]. Some of them include extensions of the MSSM.

In the bottom-up approach [46], it is found that non-universal gaugino masses with a certain ratio are favorable to improve fine-tuning in the MSSM when the messenger scale of SUSY breaking is the Planck/GUT scale. Such a favorable ratio of gaugino masses can be realized in the $\mathrm{TeV}$ scale mirage mediation [33, 34, 47, 49] and gravity mediation, e.g. moduli mediation $[43,51-53]$ and the SUSY breaking scenario, where F-components of gauge non-singlets are sizable $[42,54,59] .{ }^{1}$ On the other hand, the spectrum of the constrained MSSM with the universal gaugino mass would be unfavorable. It is also pointed out that a negative value of the stop mass squared at the Planck/GUT scale would also be favorable $[39,40]$.

Since the minimal gauge mediation [64] leads to the universal gaugino mass, that would be unfavorable from the viewpoint of fine-tuning [29,63]. Recently, Meade, Seiberg and Shih have extended the gauge mediation to general gauge mediation (GGM) [65]. (See also [66]-[77].) That leads to non-universal gaugino and soft scalar masses. Thus, it is important to study fine-tuning in the GGM. That is our purpose. ${ }^{2}$ The important difference of the gauge mediation (including GGM) from other mediation scenarios such as gravity mediation is that the messenger scale can vary from the GUT scale to a $\mathrm{TeV}$ scale and predicted A-terms are very small in most of models. These would also lead to an important difference in the fine-tuning behavior.

This paper is organized as follows. In section 2, we briefly review on the fine-tuning problem in the MSSM. Section 3 is also a brief review on the GGM. In section 4, we analyse numerically on fine-tuning in the GGM. In section 5 , we give a comment on the $\mu-B$ problem. Section 6 is devoted to conclusion.

\section{Fine tuning in the MSSM}

Here, we briefly review the fine-tuning problem in the MSSM by showing explicitly equations. In our analysis, we neglect the Yukawa couplings except the top Yukawa coupling $y_{t}$. Then, the Higgs sector in the MSSM is described as the following superpotential,

$$
W_{\mathrm{Higgs}}=\mu H_{u} H_{d}+y_{t} Q_{3} U_{3} H_{u},
$$

where $Q_{3}$, and $U_{3}$ are the chiral superfields corresponding to the left- and right-handed top quarks, respectively. The Higgs fields and top-stop multiplets as well as the gaugino fields play an important role in the fine-tuning problem. Thus, we concentrate on these fields. Their soft SUSY breaking terms are given by

$$
\begin{aligned}
V_{\text {soft }}= & m_{H_{u}}^{2}\left|H_{u}\right|^{2}+m_{H_{d}}^{2}\left|H_{d}\right|^{2}+m_{Q_{3}}^{2}\left|Q_{3}\right|^{2}+m_{U_{3}}^{2}\left|U_{3}\right|^{2} \\
& +\left(\mu B H_{u} H_{d}+y_{t} A_{t} Q_{3} U_{3} H_{u}+\text { h.c. }\right),
\end{aligned}
$$

\footnotetext{
${ }^{1}$ Those spectra with less fine-tuning also have interesting aspects on the dark matter physics [60-62].

${ }^{2}$ See also $[63,72]$.
} 
where $m_{X}\left(X=H_{u, d}, Q_{3}, U_{3}\right)$ are the soft scalar masses for $X$, respectively, $\mu B$ is the SUSY breaking mass, i.e. the so-called $B$-term. Note that we utilize the same notation for denoting a chiral superfield and its lowest scalar component.

The soft SUSY breaking mass for the up-type Higgs $m_{H_{u}}$ is subject to relatively large logarithmic radiative correction (1.1) from mainly stop loops. The radiative correction $\Delta m_{H_{u}}^{2}$ is comparable to the stop mass with the negative sign, i.e. $\Delta m_{H_{u}}^{2} \sim-m_{\tilde{t}}^{2}$. Such a large and negative correction leads to the EW symmetry breaking at the weak scale. Here, we define the averaged top squark mass $m_{\tilde{t}}$ as

$$
m_{\tilde{t}}^{2} \equiv \sqrt{m_{Q_{3}}^{2}\left(M_{Z}\right) m_{U_{3}}^{2}\left(M_{Z}\right)} .
$$

A stationary condition of the Higgs potential gives the relation among the $Z$ boson mass $M_{Z}$, the $\mu$ parameter and soft scalar masses, $m_{H_{u}}^{2}$ and $m_{H_{d}}^{2}$, as

$$
\frac{M_{Z}^{2}}{2}=-\mu^{2}\left(M_{Z}\right)-\frac{m_{H_{u}}^{2}\left(M_{Z}\right) \tan ^{2} \beta-m_{H_{d}}^{2}\left(M_{Z}\right)}{\tan ^{2} \beta-1},
$$

where $\tan \beta \equiv\left\langle H_{u}\right\rangle /\left\langle H_{d}\right\rangle$. The lightest Higgs boson mass is constrained by

$$
\begin{aligned}
m_{h}^{2} \leq & M_{Z}^{2} \cos ^{2} 2 \beta\left(1-\frac{3 m_{t}^{2}}{8 \pi^{2} v^{2}} \ln \frac{m_{\tilde{t}}^{2}}{m_{t}^{2}}\right) \\
& +\frac{3 m_{t}^{4}}{4 \pi^{2} v^{2}}\left[\ln \frac{m_{\tilde{t}}^{2}}{m_{t}^{2}}+\frac{\tilde{A}_{t}^{2}}{m_{\tilde{t}}^{2}}\left(1-\frac{\tilde{A}_{t}^{2}}{12 m_{\tilde{t}}^{2}}\right)\right. \\
& \left.+\frac{1}{16 \pi^{2}}\left(\frac{3 m_{t}^{2}}{2 v^{2}}-32 \pi \alpha_{3}\right)\left\{\frac{2 \tilde{A}_{t}^{2}}{m_{\tilde{t}}^{2}}\left(1-\frac{\tilde{A}_{t}^{2}}{12 m_{\tilde{t}}^{2}}\right) \ln \frac{m_{\tilde{t}}^{2}}{m_{t}^{2}}+\left(\ln \frac{m_{\tilde{t}}^{2}}{m_{t}^{2}}\right)^{2}\right\}\right]
\end{aligned}
$$

within the 2-loop approximation $[4,5]$, where $v=174 \mathrm{GeV}, \tilde{A}_{t} \equiv A_{t}\left(M_{Z}\right)-\mu \cot \beta$ and $m_{t}$ is the running top squark mass at $M_{Z}$.

The current experimental lower bound for the Higgs mass is given by the LEP experiment as $m_{h} \geq 114.4 \mathrm{GeV}$. In order to realize $m_{h} \geq 114.4 \mathrm{GeV}$, a large top squark mass is required as $m_{\tilde{t}} \gtrsim 1 \mathrm{TeV}$ when $\left|A_{t}\left(M_{Z}\right) / m_{\tilde{t}}\right| \lesssim 1$.0. The soft scalar mass of the up-sector Higgs field, $m_{H_{u}}$ suffers from a large radiative correction according to such a large top squark mass through (1.1). Therefore, a few percent of fine-tuning between $m_{H_{u}}^{2}$ and $\mu^{2}$ is required in (2.4) in order to realize the EW symmetry breaking with the experimentally observed $Z$ boson mass, $M_{Z} \simeq 91.2 \mathrm{GeV}$. That is the so-called little hierarchy problem. We investigate this fine-tuning problem in the context of the GGM. Furthermore, when $\left|A_{t}\left(M_{Z}\right) / m_{\tilde{t}}\right| \lesssim 1.5$, the condition $m_{h} \geq 114.4 \mathrm{GeV}$ requires $m_{\tilde{t}} \gtrsim 500 \mathrm{GeV}$. Hence, the stop mixing $A_{t} / m_{\tilde{t}}$ is important $[4,5,78]$.

\section{General gauge mediation}

Before considering the fine-tuning problem in the GGM, we also give a brief review on the GGM. Recently, Meade, Seiberg and Shih have presented the most general spectrum which can be obtained in gauge mediated SUSY breaking model [65]. A careful definition 
of gauge mediation mechanism has been given in the work, that is, in the limit that the MSSM gauge couplings $\alpha_{i} \rightarrow 0$, the theory decouples into the MSSM and a separate hidden sector which breaks SUSY. Following the convention, we label the gauge groups, SU(3), $\mathrm{SU}(2)$ and $\mathrm{U}(1)$ of the MSSM by $a=3,2,1$, respectively. Within the framework of the GGM, the three gaugino masses $M_{a}(a=1,2,3)$ of the MSSM are given at the messenger scale $M$ as,

$$
M_{a}=2 g_{a}^{2} B_{a} .
$$

In general, $B_{a}(a=1,2,3)$ are three independent complex parameters. If CP phases of $B_{a}$ are not aligned each other, that would lead to a serious CP problem. Thus, we use $B_{a}$ as three real parameters. The soft scalar masses squared are also given in the GGM as

$$
m_{\tilde{f}}^{2}=g_{1}^{2} Y_{f} \zeta+\sum_{a=1}^{3} g_{a}^{4} c_{2}(f ; a) A_{a}
$$

at $M$, where $c_{2}(f ; a)$ is the quadratic Casimir of the representation of fermion $f$ under the gauge group corresponding to the label $a$. Here, $A_{a}(a=1,2,3)$ are three independent real parameters. Hereafter, we concentrate on the models with $\zeta=0 .{ }^{3}$ In this case, there are the mass relations at $M$

$$
m_{Q_{f}}^{2}+m_{D_{f}}^{2}+m_{E_{f}}^{2}-m_{L_{f}}^{2}-2 m_{U_{f}}^{2}=0, \quad m_{H_{u}}=m_{H_{d}},
$$

where $m_{Q_{f}}, m_{U_{f}}, m_{D_{f}}, m_{L_{f}}$, and $m_{E_{f}}$ denote soft scalar masses for the $f$-th generation of the left-handed squarks, up-sector right-handed squarks, down-sector right-handed squarks, left-handed sleptons and right-handed sleptons. Thus, the $\mathrm{U}(1)_{Y} D$-term $S$, i.e.,

$$
S=m_{H_{u}}^{2}-m_{H_{d}}^{2}+\sum_{f=1}^{3}\left(m_{Q_{f}}^{2}+m_{D_{f}}^{2}+m_{E_{f}}^{2}-m_{L_{f}}^{2}-2 m_{U_{f}}^{2}\right),
$$

vanishes at the messenger scale $M$. Furthermore, its RG equation is given as

$$
(4 \pi)^{2} \frac{d S}{d t}=-b_{1} g_{1}^{2}(t) S(t)
$$

where $t \equiv 2 \log \left(M_{Z} / \bar{\mu}\right), \bar{\mu}$ is an arbitrary energy scale, and $b_{1}=33 / 5$ (and $b_{2}=1, b_{3}=-3$ for references). Thus, when $S$ is vanishing at $M$, it vanishes at any scale. For concreteness, we show explicitly the initial conditions of the soft scalar masses, $m_{Q_{3}}, m_{U_{3}}, m_{H_{u}}$ and $m_{H_{d}}$ as

$$
\begin{aligned}
m_{Q_{3}}^{2}(M) & =\frac{4}{3} g_{3}^{4}(M) A_{3}+\frac{3}{4} g_{2}^{4}(M) A_{2}+\frac{3}{5}\left(\frac{1}{6}\right)^{2} g_{1}^{4}(M) A_{1} \\
& =(4 \pi)^{4} B_{3}^{2}\left[\frac{4}{3} \tilde{\alpha}_{3}^{2}(M) a_{3}+\frac{3}{4} \tilde{\alpha}_{2}^{2}(M) a_{2}+\frac{1}{60} \tilde{\alpha}_{1}^{2}(M) a_{1}\right] \\
m_{U_{3}}^{2}(M) & =\frac{4}{3} g_{3}^{4}(M) A_{3}+\frac{3}{5}\left(-\frac{2}{3}\right)^{2} g_{1}^{4}(M) A_{1} \\
& =(4 \pi)^{4} B_{3}^{2}\left[\frac{4}{3} \tilde{\alpha}_{3}^{2}(M) a_{3}+\frac{4}{15} \tilde{\alpha}_{1}^{2}(M) a_{1}\right]
\end{aligned}
$$

\footnotetext{
${ }^{3}$ This situation, $\zeta=0$, can be realized by invoking messenger parity.
} 


$$
\begin{aligned}
m_{H_{u}}^{2}(M) & =m_{H_{d}}^{2}(M) \\
& =\frac{3}{4} g_{2}^{4}(M) A_{2}+\frac{3}{5}\left( \pm \frac{1}{2}\right)^{2} g_{1}^{4}(M) A_{1} \\
& =(4 \pi)^{4} B_{3}^{2}\left[\frac{3}{4} \tilde{\alpha}_{2}^{2}(M) a_{2}+\frac{3}{20} \tilde{\alpha}_{1}^{2}(M) a_{1}\right],
\end{aligned}
$$

where $\tilde{\alpha}_{a} \equiv \alpha_{a} /(4 \pi) \equiv g_{a}^{2} /(4 \pi)^{2}$. Here, we have defined the ratios

$$
a_{a} \equiv \frac{A_{a}}{B_{3}^{2}},
$$

for convenience. Similarly, we define the ratios of gaugino masses to the gluino mass,

$$
b_{a} \equiv \frac{B_{a}}{B_{3}} .
$$

The initial condition of the $A$-term in the GGM is given as

$$
A_{t}=0
$$

at $M$. Thus, the $A$-term $A_{t}$ at the weak scale is given only by the RG effect between the weak scale and the messenger scale $M$. This initial condition is important because the stop mixing $A_{t} / m_{\tilde{t}}$ at the weak scale has a significant effect on the Higgs mass (2.5).

By utilizing these gaugino and sfermion masses given in the GGM, we numerically analyze the fine-tuning problem in the next section.

\section{Numerical analyses}

We study the fine-tuning problem in the GGM and present numerical analyses. In gauge mediated SUSY breaking models, phenomenological consequences at the EW scale generally depend on the messenger scale $M$. We present our analyses for three typical messenger scales, that is (i) GUT scale $M=\Lambda_{\mathrm{GUT}} \equiv 2 \times 10^{16} \mathrm{GeV}$, (ii) intermediate scale $M=10^{10} \mathrm{GeV}$, and (iii) relatively low energy scale $M=10^{6} \mathrm{GeV}$.

Firstly, we give the soft parameters at the EW scale by integrating the 1-loop RG equations [6-10]. The gaugino mass at the EW scale are

$$
\begin{aligned}
& M_{1}\left(M_{Z}\right) \simeq 0.428 B_{1}, \\
& M_{2}\left(M_{Z}\right) \simeq 0.859 B_{2}, \\
& M_{3}\left(M_{Z}\right) \simeq 3.00 B_{3} .
\end{aligned}
$$

In this analysis, we use the values of gauge couplings at the EW scale as $\tilde{\alpha}_{1}\left(M_{Z}\right) \simeq$ $1.36 \times 10^{-3}, \tilde{\alpha}_{2}\left(M_{Z}\right) \simeq 2.72 \times 10^{-3}$, and $\tilde{\alpha}_{3}\left(M_{Z}\right) \simeq 9.50 \times 10^{-3}$. These couplings in the MSSM would be unified at the GUT scale within a good accuracy. In addition, we use the running top mass $m_{t}=164.5 \mathrm{GeV}$ at $M_{Z}$ and $\tan \beta=10$ for numerical analysis.

The scalar masses such as $m_{Q_{3}}, m_{U_{3}}, m_{H_{u, d}}$, and $A_{t}$, which are important to discuss the fine-tuning problem, are given for each typical messenger scale as 
(i) $M=\Lambda_{\mathrm{GUT}}$,

$$
\begin{aligned}
& m_{Q_{3}}^{2}\left(M_{Z}\right) \simeq 6.07 B_{3}^{2}-0.0120 B_{1} B_{3}-0.00754 B_{1}^{2}-0.0834 B_{2} B_{3} \\
&-0.00245 B_{1} B_{2}+0.437 B_{2}^{2} \\
&-0.116 m_{H_{u}}^{2}(M)+0.884 m_{Q_{3}}^{2}(M)-0.116 m_{U_{3}}^{2}(M), \\
& m_{U_{3}}^{2}\left(M_{Z}\right) \simeq 5.11 B_{3}^{2}-0.0240 B_{1} B_{3}+0.0495 B_{1}^{2}-0.167 B_{2} B_{3} \\
&-0.00490 B_{1} B_{2}-0.202 B_{2}^{2} \\
&-0.232 m_{H_{u}}^{2}(M)-0.232 m_{Q_{3}}^{2}(M)+0.768 m_{U_{3}}^{2}(M), \\
& m_{H_{u}}^{2}\left(M_{Z}\right) \simeq-2.90 B_{3}^{2}-0.0361 B_{1} B_{3}+0.00505 B_{1}^{2}-0.250 B_{2} B_{3} \\
&-0.00735 B_{1} B_{2}+0.235 B_{2}^{2} \\
&+0.652 m_{H_{u}}^{2}(M)-0.348 m_{Q_{3}}^{2}(M)-0.348 m_{U_{3}}^{2}(M), \\
& m_{H_{d}}^{2}\left(M_{Z}\right) \simeq 0.538 B_{2}^{2}+0.0415 B_{1}^{2}+m_{H_{d}}^{2}(M), \\
& A_{t}\left(M_{Z}\right) \simeq 2.20 B_{3}+0.278 B_{2}+0.0352 B_{1},
\end{aligned}
$$

(ii) $M=10^{10} \mathrm{GeV}$,

$$
\begin{aligned}
& m_{Q_{3}}^{2}\left(M_{Z}\right) \simeq 5.43 B_{3}^{2}-0.00327 B_{1} B_{3}-0.000940 B_{1}^{2}-0.0331 B_{2} B_{3} \\
&-0.000404 B_{1} B_{2}+0.227 B_{2}^{2} \\
&-0.0958 m_{H_{u}}^{2}(M)+0.904 m_{Q_{3}}^{2}(M)-0.0958 m_{U_{3}}^{2}(M), \\
& m_{U_{3}}^{2}\left(M_{Z}\right) \simeq 4.76 B_{3}^{2}-0.00654 B_{1} B_{3}+0.0142 B_{1}^{2}-0.0661 B_{2} B_{3} \\
&-0.000807 B_{1} B_{2}-0.0701 B_{2}^{2} \\
&-0.192 m_{H_{u}}^{2}(M)-0.192 m_{Q_{3}}^{2}(M)+0.808 m_{U_{3}}^{2}(M), \\
& m_{H_{u}}^{2}\left(M_{Z}\right) \simeq- 2.03 B_{3}^{2}-0.00981 B_{1} B_{3}+0.00405 B_{1}^{2}-0.0992 B_{2} B_{3} \\
&-0.00121 B_{1} B_{2}+0.157 B_{2}^{2} \\
&+0.712 m_{H_{u}}^{2}(M)-0.288 m_{Q_{3}}^{2}(M)-0.288 m_{U_{3}}^{2}(M), \\
& m_{H_{d}}^{2}\left(M_{Z}\right) \simeq 0.262 B_{2}^{2}+0.0103 B_{1}^{2}+m_{H_{d}}^{2}(M), \\
& A_{t}\left(M_{Z}\right) \simeq 1.93 B_{3}+0.181 B_{2}+0.0167 B_{1},
\end{aligned}
$$

(iii) $M=10^{6} \mathrm{GeV}$,

$$
\begin{aligned}
m_{Q_{3}}^{2}\left(M_{Z}\right) \simeq & 4.24 B_{3}^{2}-0.000733 B_{1} B_{3}-0.0000353 B_{1}^{2}-0.00879 B_{2} B_{3} \\
& -0.0000603 B_{1} B_{2}+0.111 B_{2}^{2} \\
& -0.0669 m_{H_{u}}^{2}(M)+0.933 m_{Q_{3}}^{2}(M)-0.0669 m_{U_{3}}^{2}(M), \\
m_{U_{3}}^{2}\left(M_{Z}\right) \simeq & 3.90 B_{3}^{2}-0.00147 B_{1} B_{3}+0.00563 B_{1}^{2}-0.0176 B_{2} B_{3} \\
& -0.000121 B_{1} B_{2}-0.0198 B_{2}^{2} \\
& -0.134 m_{H_{u}}^{2}(M)-0.134 m_{Q_{3}}^{2}(M)+0.866 m_{U_{3}}^{2}(M), \\
m_{H_{u}}^{2}\left(M_{Z}\right) \simeq & -1.03 B_{3}^{2}-0.00220 B_{1} B_{3}+0.00234 B_{1}^{2}-0.0264 B_{2} B_{3} \\
& -0.000181 B_{1} B_{2}+0.0914 B_{2}^{2} \\
& +0.799 m_{H_{u}}^{2}(M)-0.201 m_{Q_{3}}^{2}(M)-0.201 m_{U_{3}}^{2}(M),
\end{aligned}
$$




$$
\begin{aligned}
m_{H_{d}}^{2}\left(M_{Z}\right) & \simeq 0.121 B_{2}^{2}+0.00366 B_{1}^{2}+m_{H_{d}}^{2}(M), \\
A_{t}\left(M_{Z}\right) & \simeq 1.47 B_{3}+0.105 B_{2}+0.00850 B_{1} .
\end{aligned}
$$

Here, we have used the initial conditions, $A_{t}(M)=S(M)=0$. The change of RG effects between the cases (ii) and (iii) is rather drastic compared with one between (i) and (ii).

If all soft parameters are taken as the same order, $B_{a} \sim m_{X}(M)$, the averaged top squark mass is approximated for each messenger scale as

$$
m_{\tilde{t}}^{2} \sim \begin{cases}6.0 B_{3}^{2} & \text { in the case (i) } \\ 5.7 B_{3}^{2} & \text { in the case (ii) } \\ 4.8 B_{3}^{2} & \text { in the case (iii) }\end{cases}
$$

For a fixed value of $\left|A_{t}\left(M_{Z}\right) / m_{\tilde{t}}\right|$, a large value of $m_{\tilde{t}}^{2}$ would be favorable to realize the Higgs mass $m_{h} \geq 114.4 \mathrm{GeV}$. That implies that a higher messenger scale would be favorable for a fixed value of the gluino mass, i.e. $B_{3}$. In order to satisfy the experimental bound for the Higgs mass, the lower bound for $B_{3}$ is roughly estimated as

$$
B_{3} \gtrsim\left\{\begin{array}{l}
200(410) \mathrm{GeV} \text { for }\left|A_{t}\left(M_{Z}\right) / m_{\tilde{t}}\right| \lesssim 1.5(1.0) \text { in the case (i) } \\
210(420) \mathrm{GeV} \text { for }\left|A_{t}\left(M_{Z}\right) / m_{\tilde{t}}\right| \lesssim 1.5(1.0) \text { in the case (ii) } \\
230(460) \mathrm{GeV} \text { for }\left|A_{t}\left(M_{Z}\right) / m_{\tilde{t}}\right| \lesssim 1.5 \text { (1.0) in the case (iii) }
\end{array}\right.
$$

Furthermore, we can estimate the stop mixing $\left|A_{t}\left(M_{Z}\right) / m_{\tilde{t}}\right|$. For example, for $B_{a} \sim$ $m_{X}(M)$ we estimate

$$
\left|A_{t}\left(M_{Z}\right) / m_{\tilde{t}}\right| \sim \begin{cases}1.0 & \text { in the case (i) } \\ 0.89 & \text { in the case (ii) } \\ 0.72 & \text { in the case (iii) }\end{cases}
$$

A large value of $\left|A_{t}\left(M_{Z}\right) / m_{\tilde{t}}\right|$ would be favorable to realize the Higgs mass $m_{h} \geq 114.4 \mathrm{GeV}$. That implies that a higher messenger scale would be favorable.

On the other hand, the dominant part of the RG effects in $m_{H_{d}}^{2}(4.6),(4.11)$ and (4.16) is due to the gluino mass, i.e. $B_{3}^{2}$. If $B_{3} \sim 500 \mathrm{GeV}$, we need fine-tuning between $m_{H_{u}}^{2}$ and $\mu^{2}$ to realize $M_{Z}$. The absolute value of coefficient of $B_{3}^{2}$ in $m_{H_{d}}^{2}\left(M_{Z}\right)$ decreases as the messenger scale $M$ decreases. Thus, for a fixed value of $B_{3}$, the degree of fine-tuning is reduced as the messenger scale becomes lower.

Thus, the tension between the fine-tuning and the lower bound of the Higgs mass $m_{h} \geq 114.4 \mathrm{GeV}$ depends non-trivially on the messenger scale $M$. Also that would depend on ratios among gaugino masses and scalar masses, although we have used $B_{a} \sim m_{X}(M)$ in the above estimation.

Toward the numerical analyses of the fine-tuning problem, we introduce fine-tuning parameters [13-18],

$$
\Delta_{Y} \equiv \frac{1}{2} \frac{Y}{M_{Z}^{2}} \frac{\partial M_{Z}^{2}}{\partial Y}
$$

which indicates that we need $100 / \Delta_{Y}$ percent of fine-tuning for $Y$ to derive $M_{Z}$. A larger value of $\Delta_{Y}$ means more severe fine-tuning to be required. 
If $B_{a}$ and $A_{a}$ are independent of each other, fine-tuning for $B_{3}$ would be most severe, because $m_{H_{d}}^{2}\left(M_{Z}\right)$ depends dominantly on $B_{3}$. For example, we can calculate

(i) $M=\Lambda_{\mathrm{GUT}}$

$$
\Delta_{B_{3}}=5.85 \hat{M}_{3}^{2}+\left(0.0364 \hat{M}_{1}+0.253 \hat{M}_{2}\right) \hat{M}_{3}
$$

(ii) $M=10^{10} \mathrm{GeV}$

$$
\Delta_{B_{3}}=4.10 \hat{M}_{3}^{2}+\left(0.00990 \hat{M}_{1}+0.100 \hat{M}_{2}\right) \hat{M}_{3},
$$

(iii) $M=10^{6} \mathrm{GeV}$

$$
\Delta_{B_{3}}=2.08 \hat{M}_{3}^{2}+\left(0.00222 \hat{M}_{1}+0.0266 \hat{M}_{2}\right) \hat{M}_{3}
$$

where $\hat{M}_{a} \equiv B_{a} / M_{Z}$. It is found that the coefficients of the terms become small as the messenger scale becomes lower. If $\Delta_{B_{3}} \leq 10$ is required under the condition $B_{1}=B_{2}=B_{3}$, the allowed value of $B_{3}$ are (i) $B_{3} \leq 110 \mathrm{GeV}$, (ii) $B_{3} \leq 140 \mathrm{GeV}$, and (iii) $B_{3} \leq 190 \mathrm{GeV}$. They could not satisfy the bounds on the Higgs mass (4.20). On the other hand, when we take $B_{3} \simeq 500 \mathrm{GeV}$, we find that severe fine-tunings such as (i) $\Delta_{B_{3}} \simeq 200$, (ii) $\Delta_{B_{3}} \simeq 140$, (iii) $\Delta_{B_{3}} \simeq 70$ are needed.

We have assumed that $B_{a}$ and $A_{a}$ are independent of each other. However, in a definite theory, they are not independent, but certain ratios are predicted in each theory. That is, in a definite theory there is one parameter, which determines the overall size of soft SUSY breaking terms. We choose $B_{3}$ as such a parameter and the ratios $a_{a}$ and $b_{a}$ are fixed in a theory. Then, we consider the fine-tuning only for $B_{3}$, i.e. $\Delta_{B_{3}}$ under fixed ratios of $a_{a}$ and $b_{a}$. Varying $a_{a}$ and $b_{a}$ means that we compare different theories in the theory space of the GGM. Then, the fine-tuning parameter can be rewritten as

(i) $M=\Lambda_{\mathrm{GUT}}$

$$
\begin{gathered}
\Delta_{B_{3}}=\hat{M}_{3}^{2}\left(5.85+0.506 b_{2}-0.465 b_{2}^{2}+0.508 a_{3}-0.122 a_{2}+0.0728 b_{1}+0.0148 b_{1} b_{2}\right. \\
\left.-0.00936 b_{1}^{2}+0.00132 a_{1}\right),
\end{gathered}
$$

(ii) $M=10^{10} \mathrm{GeV}$

$$
\begin{gathered}
\Delta_{B_{3}}=\hat{M}_{3}^{2}\left(4.10+0.200 b_{2}-0.311 b_{2}^{2}+0.825 a_{3}-0.143 a_{2}+0.0198 b_{1}+0.00245 b_{1} b_{2}\right. \\
\left.-0.00798 b_{1}^{2}-0.00495 a_{1}\right),
\end{gathered}
$$

(iii) $M=10^{6} \mathrm{GeV}$

$$
\begin{gathered}
\Delta_{B_{3}}=\hat{M}_{3}^{2}\left(2.08+0.0533 b_{2}-0.182 b_{2}^{2}+1.04 a_{3}-0.183 a_{2}+0.00444 b_{1}+0.000365 b_{1} b_{2}\right. \\
\left.-0.00465 b_{1}^{2}-0.00821 a_{1}\right) .
\end{gathered}
$$


Coefficients of $b_{1}$ and $a_{1}$ in the above equations are very small. Thus, those terms would not be important unless $b_{1}=\mathcal{O}(10)$ or $a_{1}=\mathcal{O}(100)$. Therefore, we concentrate on others and throughout our numerical analyses we take $b_{1}=a_{1}=1$ as a typical value. It is found that the coefficients of $a_{2}$ and $b_{2}^{2}$, which determines the wino mass, are negative. Hence, it would be favorable to cancel the dominant term by relatively large $b_{2}$ and/or $a_{2}$. That is, models satisfying

(i) $M=\Lambda_{\mathrm{GUT}}$

$$
\left|5.85+0.506 b_{2}-0.465 b_{2}^{2}+0.508 a_{3}-0.122 a_{2}\right| \ll 1
$$

(ii) $M=10^{10} \mathrm{GeV}$

$$
\left|4.10+0.200 b_{2}-0.311 b_{2}^{2}+0.825 a_{3}-0.143 a_{2}\right| \ll 1
$$

(iii) $M=10^{6} \mathrm{GeV}$

$$
\left|2.08+0.0533 b_{2}-0.182 b_{2}^{2}+1.04 a_{3}-0.183 a_{2}\right| \ll 1
$$

would be interesting in the theory space. For fixed values of $a_{2}$ and $a_{3}$, a favorable value of $b_{2}$ is determined. That means a favorable ratio between the gluino and wino masses such as ref. [46]. For a fixed value of $b_{2}$, a linear correlation between $a_{2}$ and $a_{3}$ is required. On the other hand, for a fixed value of $a_{2}\left(a_{3}\right)$ a quadratic relation between $b_{2}$ and $a_{3}\left(a_{2}\right)$ is required.

The results of numerical analyses are shown in figures 1-4. Figure 1 (a) and (b) show three curves corresponding to $\Delta_{B_{3}}=5,10,15$ for $B_{3}=500$ and $300 \mathrm{GeV}$ in the case (i), respectively. The darkest (darker) solid lines correspond $\Delta_{B_{3}}=5$ (10). The dotted (red) curve is $m_{h}=114.4$. GeV and the shaded (yellow) region corresponds to the region with $m_{h} \geq 114.4 \mathrm{GeV}$. In these figures, we fix $b_{1}=a_{1}=1$ and $b_{2} \simeq 4.19$ for (a) and $b_{2} \simeq 4.01$ for (b). These values of $b_{2}$ lead to $\Delta_{B_{3}}=10$ when $b_{1}=a_{1}=a_{2}=a_{3}=1 .{ }^{4}$ These figures mean how much stable the region with $\Delta_{B_{3}}=10$ is in the $\left(a_{2}, a_{3}\right)$ plane, when $b_{2}$ is fixed such that $\Delta_{B_{3}}=10$ is realized for $b_{1}=a_{1}=a_{2}=a_{3}=1$. We find from figure 1 (a) that $a_{2} \lesssim 5$ and $a_{3} \lesssim 2$ are required to realize $\Delta_{B_{3}} \sim 10$. Figure 1 (b) shows that these upper bounds of both $a_{2}$ and $a_{3}$ are raised for $B_{3}=300 \mathrm{GeV}$. It is seen from (4.26) that the widths among three lines become wider as the $B_{3}$ becomes lower. The lower bound for $a_{2}$ and $a_{3}$ are evaluated as $\left(a_{2}, a_{3}\right) \gtrsim(-45,-10)$ for $B_{3}=500 \mathrm{GeV}$ and $(-40,-9)$ for $300 \mathrm{GeV}$. These results are insensitive to a value of $a_{1}$, even if $a_{1}$ is larger such as $a_{1} \sim \mathcal{O}(10)$. Figure 1 (c) and (d) correspond to the case of $\left(b_{2}, B_{3}\right)=(1,500 \mathrm{GeV})$ and $\left(b_{2}, B_{3}\right)=(1,300 \mathrm{GeV})$, respectively. The lower bounds for $a_{2}$ are raised to $a_{2} \gtrsim-10$. It can be also found that the favorable region is $a_{3} \gtrsim-15$.

Figures 2 and 3 show the results of the same analyses as case (i) for the cases (ii) and (iii), respectively, but (a) and (c) for $B_{3}=1 \mathrm{TeV}$ and (b) and (d) for $B_{3}=500 \mathrm{GeV}$

\footnotetext{
${ }^{4}$ There is another value of $b_{2}$, which is negative and its absolute value is similar for positive one, to lead to $\Delta_{B_{3}}=10$ when $b_{1}=a_{1}=a_{2}=a_{3}=1$. In this work, we focus on only a positive value of solution but our results are not modified for a negative one.
} 


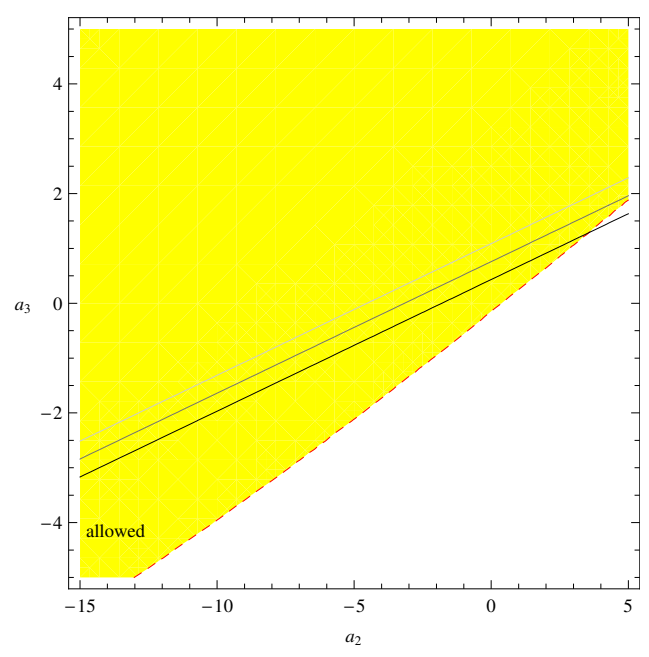

(a)

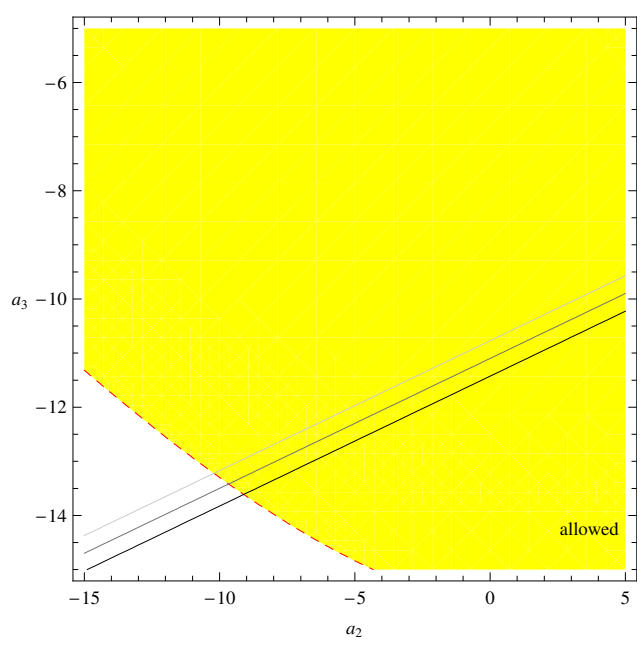

(c)

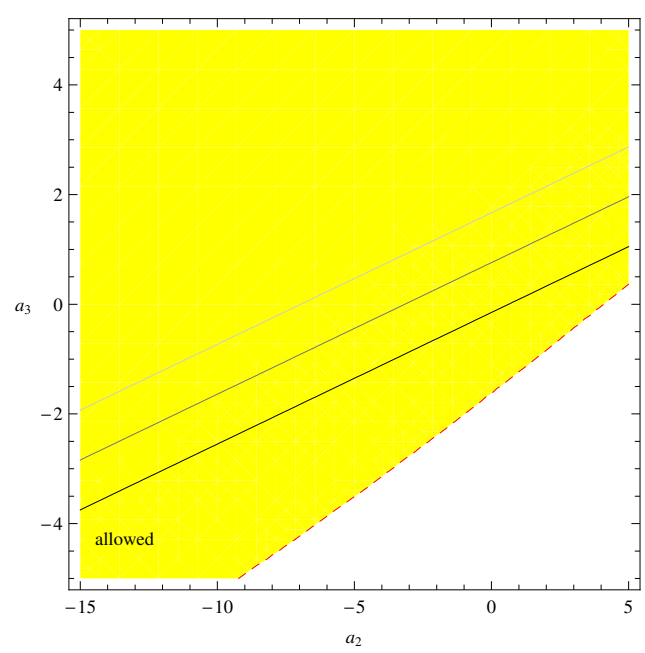

(b)

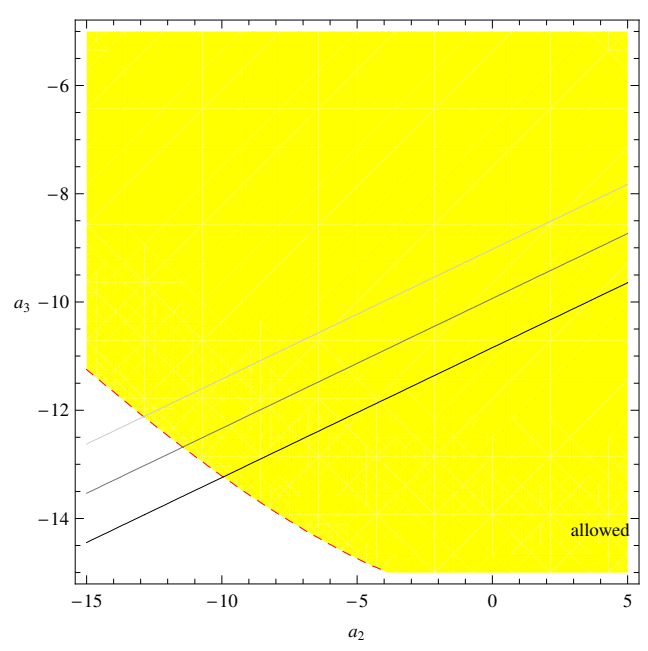

(d)

Figure 1. Lines and curve for the case (i) determined by constraints from $\Delta_{B_{3}}=5,10,15$ (solid lines), $m_{h}=114.4 \mathrm{GeV}$ (dashed (red) curve), and experimentally allowed region $m_{h} \geq 114.4 \mathrm{GeV}$ (shaded (yellow) region). The darker and darkest solid lines correspond $\Delta_{B_{3}}=10$ and 5 , respectively. We take as $b_{1}=a_{1}=1$ in all figures. (a) for $B_{3}=500 \mathrm{GeV}$ and $b_{2} \simeq 4.19$. (b) for $B_{3}=300 \mathrm{GeV}$ and $b_{2} \simeq 4.01$. These values of $b_{2}$ lead to $\Delta_{B_{3}}=10$ when $b_{1}=a_{1}=a_{2}=a_{3}=1$ in each value of $B_{3}$. (c) for $B_{3}=500 \mathrm{GeV}$ and $b_{2}=1$. (b) for $B_{3}=300 \mathrm{GeV}$ and $b_{2}=1$.

in figure 3. For the messenger scale of $M=10^{6} \mathrm{GeV}$, there is no region corresponding to $\Delta_{B_{3}} \sim 10$ and $m_{h} \geq 114.4 \mathrm{GeV}$ when the gluino mass is relatively light such as $B_{3} \sim 300 \mathrm{GeV}$. Figure 4 corresponds to the enlargement of figure 3 . All favorable regions shown in figures also satisfy the experimental bound of the top squark mass, $m_{\tilde{t}_{1}} \geq 95.7 \mathrm{GeV}$. The allowed regions become generally narrow as the messenger scale becomes lower. Especially, the values of $a_{2}$ and $a_{3}$ are constrained to only negative values 


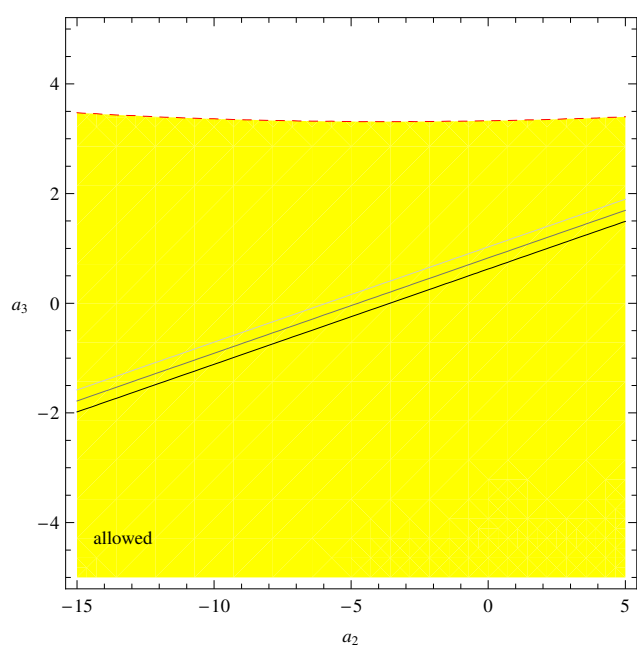

(a)

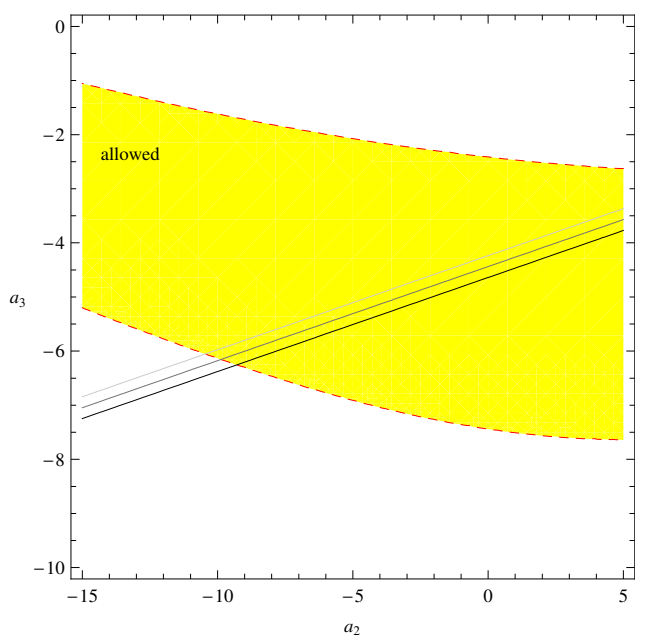

(c)

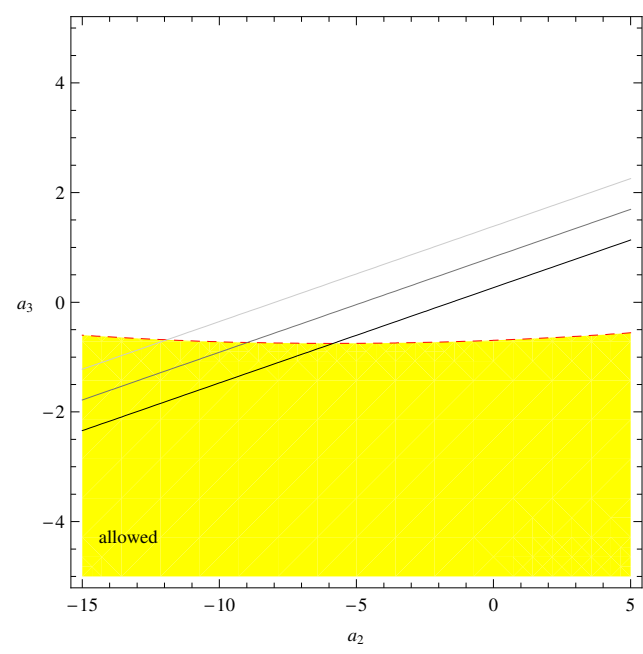

(b)

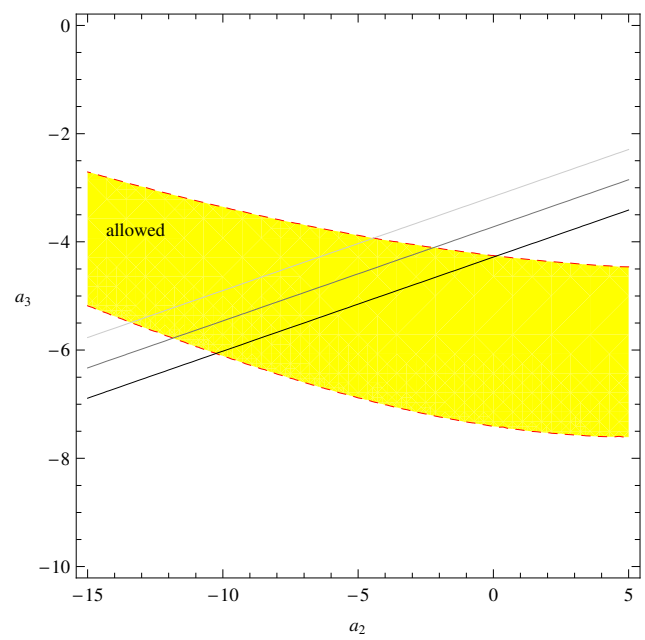

(d)

Figure 2. The same lines and curve as figure 1 but in the case (ii)

for $\left(M, B_{3}, b_{2}\right)=\left(10^{6} \mathrm{GeV}, 500 \mathrm{GeV}, 1\right)$ shown in figure $3(\mathrm{~d})$. This means that tachyonic scalar masses are required at the messenger scale to reduce the fine-tuning in the context of the GGM.

Toward for future model building of the GGM to relax the fine-tuning problem, we present a summary of a typical parameter space in tables $1,2,3$. When we fix as $a_{3}=$ 1 , which is always allowed in all cases of the messenger scale, the favorable regions are obtained as 


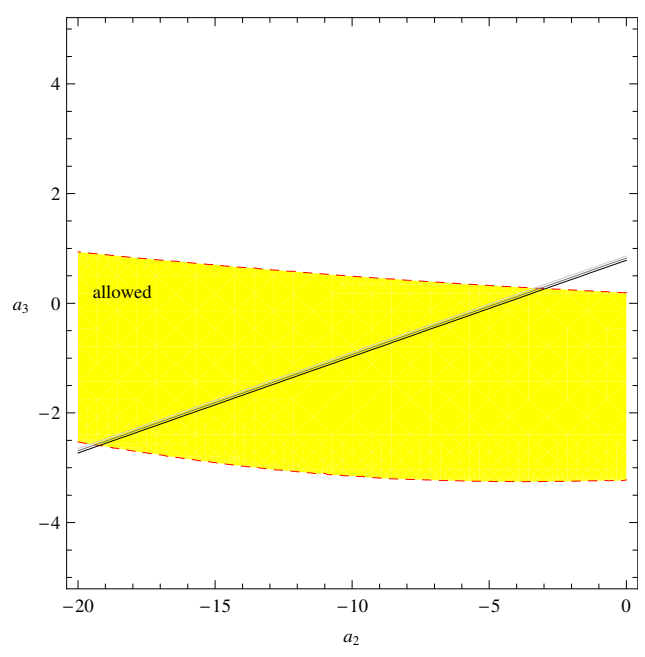

(a)

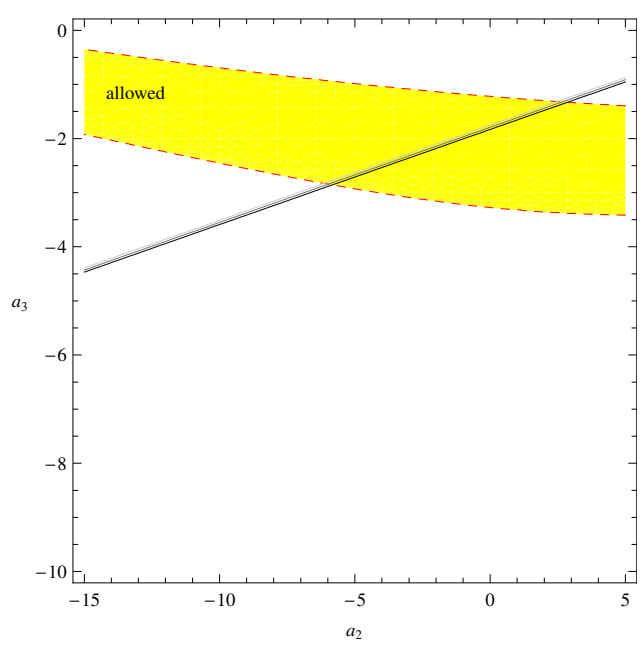

(c)

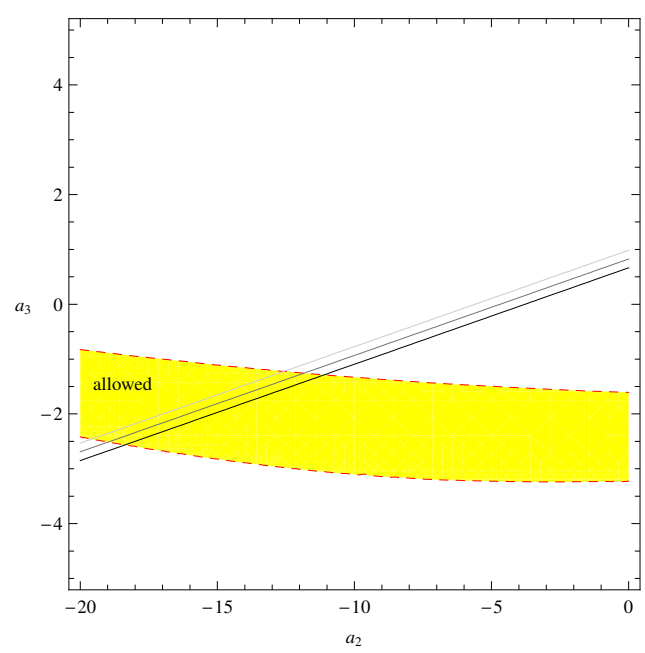

(b)

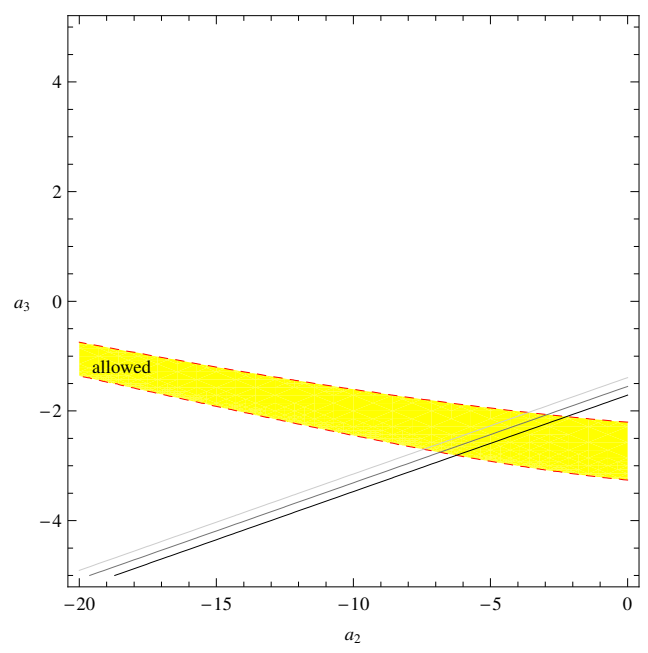

(d)

Figure 3. The same lines and curve as figure 1 but (a) and (c) for $B_{3}=1 \mathrm{TeV}$ and (b) and (d) for $B_{3}=500 \mathrm{GeV}$ in the case (iii).

(i) $M=\Lambda_{\mathrm{GUT}}$

$$
\begin{aligned}
& 0 \lesssim b_{2} \lesssim 7 \text { for }-100 \lesssim a_{2} \lesssim 40 \text { and } B_{3}=500 \mathrm{GeV} \\
& 0 \lesssim b_{2} \lesssim 6.5 \text { for }-100 \lesssim a_{2} \lesssim 40 \text { and } B_{3}=300 \mathrm{GeV}
\end{aligned}
$$

(ii) $M=10^{10} \mathrm{GeV}$

$$
\begin{aligned}
& 2 \lesssim b_{2} \lesssim 8 \text { for } a_{3}=1,-100 \lesssim a_{2} \lesssim 20, \text { and } B_{3}=500 \mathrm{GeV}, \\
& 0 \lesssim b_{2} \lesssim 6.5 \text { for } a_{3}=1,-100 \lesssim a_{2} \lesssim-10, \text { and } B_{3}=300 \mathrm{GeV}
\end{aligned}
$$




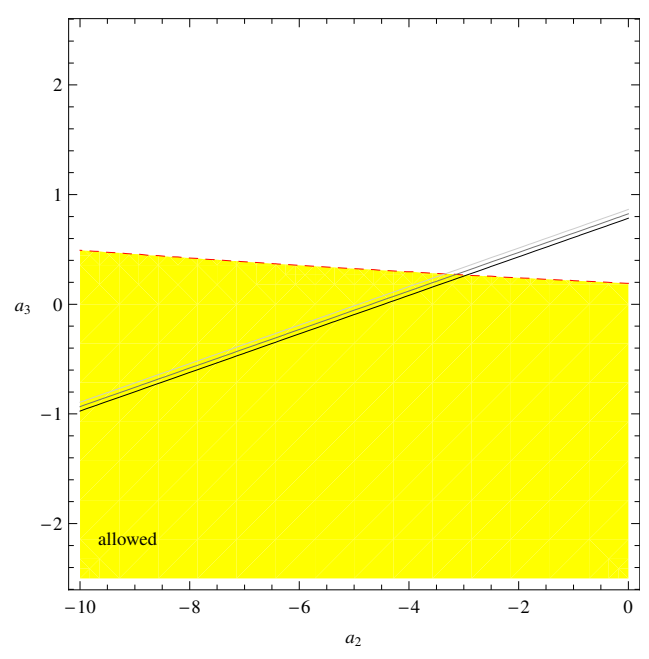

(a)

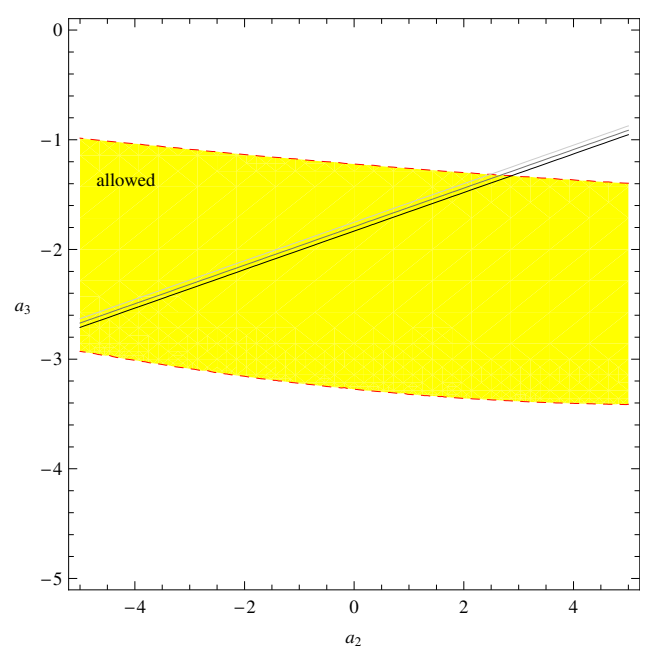

(c)

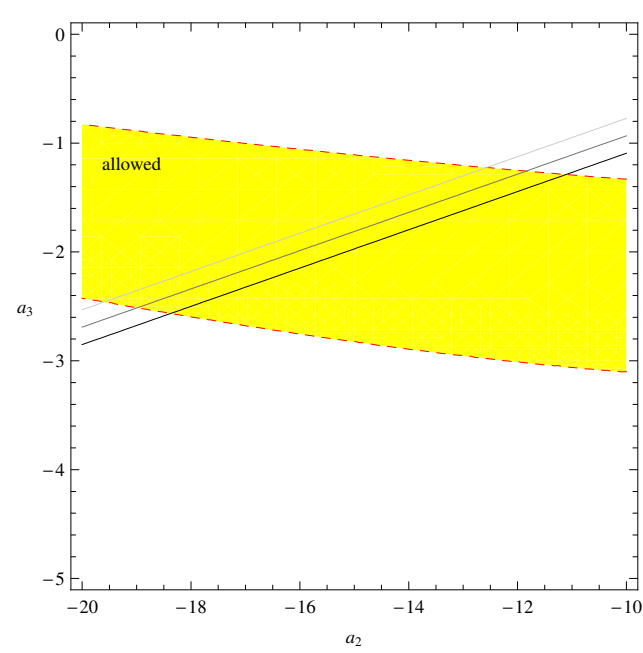

(b)

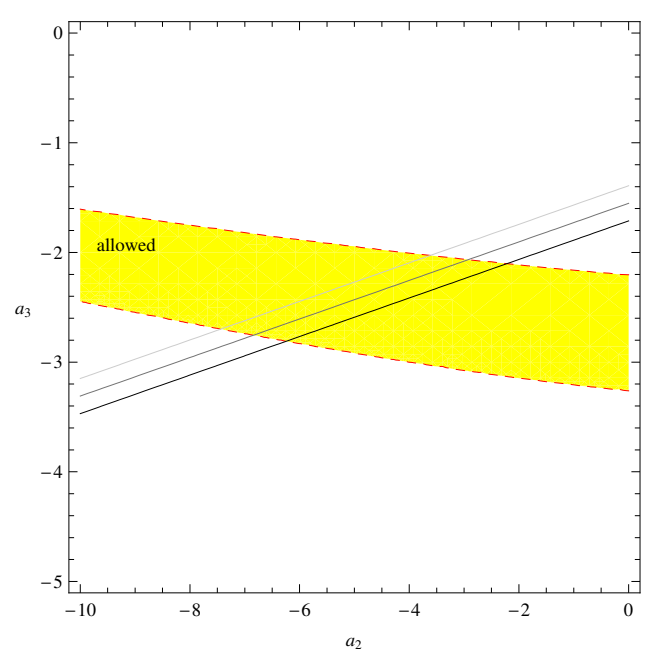

(d)

Figure 4. The enlargements of figure 3.

(iii) $M=10^{6} \mathrm{GeV}$

$5 \lesssim b_{2} \lesssim 11$ for $a_{3}=1, \quad-100 \lesssim a_{2} \lesssim 5$, and $B_{3}=1000 \mathrm{GeV}$,

$5 \lesssim b_{2} \lesssim 10$ for $a_{3}=-1,-100 \lesssim a_{2} \lesssim-10$, and $B_{3}=500 \mathrm{GeV}$.

Our results show that a certain ratio between the gluino mass and wino mass is favorable. Also, the tachyonic initial condition for stop masses at the messenger scale would be favorable, in particular in the low messenger scale scenario. For $M<10^{6} \mathrm{GeV}$, the favorable region corresponds to only negative values of both $a_{2}$ and $a_{3}$. The $A$-term $A_{t}$ plays a role in this result. Its initial value vanishes at $M$, i.e. $A_{t}(M)=0$, and its value at $M_{Z}$ is generated by RG effect as eqs. (4.8),(4.13),(4.18), which are determined mainly by 


\begin{tabular}{|c|c|c|c|c|}
\hline$B_{3}[\mathrm{GeV}]$ & $b_{2}$ & $a_{2}$ & $a_{3}$ & Figure \\
\hline 500 & 4.19 & $-45 \lesssim a_{2} \lesssim 5$ & $-10 \lesssim a_{3} \lesssim 2$ & 2 (a) \\
\hline 300 & 4.01 & $-40 \lesssim a_{2} \lesssim 10$ & $-9 \lesssim a_{3} \lesssim 5$ & 2 (b) \\
\hline 500 & 1 & $-10 \lesssim a_{2} \lesssim 50$ & $-15 \lesssim a_{3} \lesssim 0$ & 2 (c) \\
\hline 300 & 1 & $-10 \lesssim a_{2} \lesssim 50$ & $-12 \lesssim a_{3} \lesssim 3$ & $2(\mathrm{~d})$ \\
\hline
\end{tabular}

Table 1. Favorable parameter regions for (i) $M=\Lambda_{\mathrm{GUT}}$.

\begin{tabular}{|c|c|c|c|c|}
\hline$B_{3}[\mathrm{GeV}]$ & $b_{2}$ & $a_{2}$ & $a_{3}$ & Figure \\
\hline 500 & 4.12 & $-35 \lesssim a_{2} \lesssim 15$ & $-5 \lesssim a_{3} \lesssim 0$ & $3(\mathrm{a})$ \\
\hline 300 & 3.86 & $-35 \lesssim a_{2} \lesssim-10$ & $-5 \lesssim a_{3} \lesssim-1$ & 3 (b) \\
\hline 500 & 1 & $-10 \lesssim a_{2} \lesssim 10$ & $-6 \lesssim a_{3} \lesssim-3$ & 3 (c) \\
\hline 300 & 1 & $-12 \lesssim a_{2} \lesssim-2$ & $-5 \lesssim a_{3} \lesssim-4$ & 3 (d) \\
\hline
\end{tabular}

Table 2. Favorable parameter regions for (ii) $M=10^{10} \mathrm{GeV}$.

\begin{tabular}{|c|c|c|c|c|}
\hline$B_{3}[\mathrm{GeV}]$ & $b_{2}$ & $a_{2}$ & $a_{3}$ & Figure \\
\hline 1000 & 4.10 & $-20 \lesssim a_{2} \lesssim-5$ & $-2 \lesssim a_{3} \lesssim 0$ & 4 (a) \\
\hline 500 & 3.93 & $-20 \lesssim a_{2} \lesssim-10$ & $-2 \lesssim a_{3} \lesssim-1$ & 4 (b) \\
\hline 1000 & 1 & $-5 \lesssim a_{2} \lesssim 5$ & $-2 \lesssim a_{3} \lesssim-1$ & 4 (c) \\
\hline 500 & 1 & $-5 \lesssim a_{2} \lesssim-3$ & $-3 \lesssim a_{3} \lesssim-2$ & 4 (d) \\
\hline
\end{tabular}

Table 3. Favorable parameter regions for (ii) $M=10^{6} \mathrm{GeV}$.

$B_{3}$ and $B_{2}$. However, a value of $\left|A_{t}\left(M_{Z}\right)\right|$ at $M_{Z}$ is smaller as the messenger scale becomes lower, because the RG effects become smaller. On the other hand, a large value of the stop mixing $\left|A_{t} / m_{\tilde{t}}\right|$ is favorable to increase the Higgs mass, $m_{h}$. Thus, if a value of $\left|A_{t}\left(M_{Z}\right)\right|$ is small, we have to decrease a value $m_{\tilde{t}}$ to obtain a large stop mixing $\left|A_{t} / m_{\tilde{t}}\right|$. That can be realized by imposing the tachyonic initial condition of the stop mass at $M$.

We also give the mass spectra of gluino, wino, and stop for typical parameters of the favorable regions in table 4 . We find that the smallest masses of wino and stop are realized in the case (i) with $B_{3}=300 \mathrm{GeV}, a_{3}=-1$, and $a_{2}=30$ as $M_{2} \simeq 517 \mathrm{GeV}$ and $m_{\tilde{t}} \simeq 555 \mathrm{GeV}$. On the other hand, the largest masses of wino and stop are given in the case (iii) with $B_{3}=10^{3} \mathrm{GeV}, a_{3}=1$ and $a_{2}=-50$ as $M_{2} \simeq 7150 \mathrm{GeV}$ and $m_{\tilde{t}} \simeq 2420 \mathrm{GeV}$.

\section{$5 \quad \mu-B$ problem}

Here, we comment on the $\mu$-term and $B$-term. How to generate the $\mu$-term and $B$-term is another important issue. Within the framework of the gauge mediation, a simple mechanism to generate the $\mu$-term would lead to

$$
\frac{\mu B}{\mu^{2}}=\mathcal{O}\left(16 \pi^{2}\right)
$$




\begin{tabular}{|c|c|c|c|c|c|c|c|}
\hline$M[\mathrm{GeV}]$ & $B_{3}[\mathrm{GeV}]$ & $a_{3}$ & $a_{2}$ & $b_{2}$ & $M_{3}[\mathrm{GeV}]$ & $M_{2}[\mathrm{GeV}]$ & $m_{\tilde{t}}[\mathrm{GeV}]$ \\
\hline $2 \times 10^{16}$ & 500 & 1 & -50 & 5.71 & 1500 & 2450 & 1180 \\
\hline $2 \times 10^{16}$ & 500 & -1 & -50 & 4.22 & 1500 & 1810 & 863 \\
\hline $2 \times 10^{16}$ & 500 & -1 & -1 & 1.31 & 1500 & 563 & 865 \\
\hline $2 \times 10^{16}$ & 500 & -1 & 40 & 1.42 & 1500 & 609 & 803 \\
\hline $2 \times 10^{16}$ & 300 & 1 & -50 & 5.59 & 900 & 1440 & 722 \\
\hline $2 \times 10^{16}$ & 300 & 1 & 1 & 4.01 & 900 & 1030 & 616 \\
\hline $2 \times 10^{16}$ & 300 & 1 & 30 & 2.63 & 900 & 677 & 522 \\
\hline $2 \times 10^{16}$ & 300 & -1 & -50 & 5.36 & 900 & 1380 & 704 \\
\hline $2 \times 10^{16}$ & 300 & -1 & 1 & 3.67 & 900 & 947 & 615 \\
\hline $2 \times 10^{16}$ & 300 & -1 & 30 & 2.01 & 900 & 517 & 555 \\
\hline $10^{10}$ & 500 & 1 & -50 & 6.48 & 1500 & 2790 & 1280 \\
\hline $10^{10}$ & 500 & 1 & 1 & 4.12 & 1500 & 1770 & 1230 \\
\hline $10^{10}$ & 500 & 1 & 10 & 3.58 & 1500 & 1520 & 1210 \\
\hline $10^{10}$ & 500 & -1 & -50 & 6.04 & 1500 & 2590 & 1150 \\
\hline $10^{10}$ & 500 & -1 & 1 & 3.34 & 1500 & 1440 & 1110 \\
\hline $10^{10}$ & 500 & -1 & 10 & 2.55 & 1500 & 1100 & 1100 \\
\hline $10^{10}$ & 300 & 1 & -30 & 5.50 & 900 & 1420 & 755 \\
\hline $10^{10}$ & 300 & -1 & -50 & 5.89 & 900 & 1510 & 682 \\
\hline $10^{10}$ & 300 & -1 & -10 & 3.83 & 900 & 987 & 669 \\
\hline $10^{6}$ & 1000 & 1 & -50 & 8.32 & 3000 & 7150 & 2420 \\
\hline $10^{6}$ & 1000 & -1 & -50 & 7.59 & 3000 & 6520 & 1800 \\
\hline $10^{6}$ & 1000 & -1 & 1 & 2.21 & 3000 & 1900 & 1800 \\
\hline $10^{6}$ & 500 & -1 & -30 & 5.97 & 1500 & 2570 & 897 \\
\hline
\end{tabular}

Table 4. Mass spectra of gluino, wino, and stop in typical parameter space.

This ratio would cause a problem if

$$
\mu^{2} \sim m_{H_{u}}^{2}\left(M_{Z}\right), m_{H_{d}}^{2}\left(M_{Z}\right) .
$$

When both (5.1) and (5.2) hold, we could not realize the successful EW symmetry breaking. That is often called the $\mu-B$ problem of the gauge mediation.

However, in the previous section, we have studied models with spectra different from eq. (5.2). From the viewpoint of fine-tuning between $\mu^{2}$ and $m_{H_{u}}^{2}\left(M_{Z}\right)$, the favorable spectrum is that $\mu,\left|m_{H_{u}}\left(M_{Z}\right)\right|=\mathcal{O}(100) \mathrm{GeV}$ and other SUSY breaking masses are of order of a few $\mathrm{TeV}$. Indeed, if we can obtain the following hierarchy,

$$
\mu^{2} \sim m_{H_{u}}^{2} \ll \mu B \ll m_{H_{d}}^{2},
$$

we can realize the successful EW symmetry breaking. It has been already pointed out in [79] that the above hierarchy would be favorable in the gauge mediation. Also, such a pattern has been studied within the framework of the $\mathrm{TeV}$ scale mirage scenario [34], i.e. the mass pattern II. 
This pattern of hierarchy can be realized in our analyses. A relatively large $B_{2}$ is favorable to obtain a large $m_{H_{d}}$ seen as in (4.7), (4.12), and (4.17). For example, if we take $M=10^{6} \mathrm{GeV}, B_{3}=1 \mathrm{TeV}, a_{3}=1, a_{2}=-50$, and $b_{2} \simeq 8.32$, which lead to $\Delta_{B_{3}}=10$, $M_{3} \simeq 3 \mathrm{TeV}$, and $M_{2} \simeq 7.15 \mathrm{TeV}$, and $m_{\tilde{t}}=2.42 \mathrm{TeV}$, we obtain

$$
m_{H_{d}}^{2}\left(M_{Z}\right) \simeq 2.89^{2} \mathrm{TeV}^{2}
$$

By using

$$
\sin 2 \beta=\frac{2 \mu B}{2|\mu|^{2}+m_{H_{u}}^{2}+m_{H_{d}}^{2}}
$$

with $\tan \beta=10$, the above value of $m_{H_{d}}^{2}\left(M_{Z}\right) \simeq 2.89^{2} \mathrm{TeV}^{2}$ determines the value of $\mu B$ as

$$
\mu B \simeq 911^{2} \mathrm{GeV}^{2} .
$$

That is, we have $\mu B / \mu^{2}=\mathcal{O}(100)$ for $\mu \sim 100 \mathrm{GeV}$. Such a ratio $\mu B / \mu^{2}$ could be realized by a simple mechanism to generate the $\mu$-term and $B$-term $(5.1) .{ }^{5}$ Therefore, this parameter set, which relaxes the fine-tuning problem, would also be favorable from the viewpoint of the $\mu-B$ problem.

\section{Summary}

We have studied the fine-tuning problem in the context of general gauge mediation. Numerical analyses toward for relaxing the fine-tuning in the problem have been presented. We analysed the problem in typical three cases of the messenger scale, that is, GUT $\left(2 \times 10^{16} \mathrm{GeV}\right)$, intermediate $\left(10^{10} \mathrm{GeV}\right)$, and relatively low energy $\left(10^{6} \mathrm{GeV}\right)$ scales. In each case, the parameter space with less fine-tuning such as $10 \%$ has been found. It has also been shown that the favorable region becomes narrow as the messenger scale becomes lower, especially, $-10 \lesssim a_{2} \lesssim 50$ and $-15 \lesssim a_{3} \lesssim 0$ are allowed for $B_{3}=500 \mathrm{GeV}$ and $b_{1}=b_{2}=a_{1}=1$ in the case (i), $-10 \lesssim a_{2} \lesssim 10$ and $-6 \lesssim a_{3} \lesssim-3$ for $B_{3}=500 \mathrm{GeV}$ and $b_{1}=b_{2}=a_{1}=1$ in the case (ii), and $-5 \lesssim a_{2} \lesssim-3$ and $-3 \lesssim a_{3} \lesssim-2$ for $B_{3}=500 \mathrm{GeV}$ and $b_{1}=b_{2}=a_{1}=1$ in the case (iii). Our results imply that certain ratios between the gluino and wino masses as well as scalar masses are favorable to relax the fine-tuning problem. Also, tachyonic initial conditions of scalar masses are favored, in particular in the relatively low messenger scale scenario. Furthermore, the type of spectra with $\mu \approx 100 \mathrm{GeV}$ and a few TeV of other SUSY breaking masses is also favorable from the viewpoint of the $\mu-B$ problem. Thus, it would be important to construct explicit models, which realize certain ratios among gaugino and scalar masses.

Note to be added. While this paper was being completed, ref. [80] appeared, where also fine tuning in the GGM was studied.

\footnotetext{
${ }^{5}$ In this example, we use the large ratio of $\left|a_{2} / a_{3}\right|$, i.e. $a_{3}=1$ and $a_{2}=-50$, but realization of such a ratio may not be straightforward in explict model building. As another example, we take $M=10^{10} \mathrm{GeV}$, $B_{3}=500 \mathrm{GeV}, a_{3}=1, a_{2}=1$, and $b_{2} \simeq 4.12$. This example leads to $m_{H_{d}}\left(M_{Z}\right) \simeq 1.06 \mathrm{TeV}$ and $\mu B / \mu^{2}=\mathcal{O}(10)$. That would lead to the above hierarchy (5.3) although the gap of hierarchy would be smaller than the first example.
} 


\section{Acknowledgments}

T. K. is supported in part by the Grant-in-Aid for Scientific Research No. 20540266 from the Ministry of Education, Culture, Sports, Science and Technology of Japan. T. K. and R. T. are also supported in part by the Grant-in-Aid for the Global COE Program "The Next Generation of Physics, Spun from Universality and Emergence" from the Ministry of Education, Culture,Sports, Science and Technology of Japan.

Open Access. This article is distributed under the terms of the Creative Commons Attribution Noncommercial License which permits any noncommercial use, distribution, and reproduction in any medium, provided the original author(s) and source are credited.

\section{References}

[1] Y. Okada, M. Yamaguchi and T. Yanagida, Renormalization group analysis on the Higgs mass in the softly broken supersymmetric standard model, Phys. Lett. B 262 (1991) 54 [SPIRES].

[2] H.E. Haber and R. Hempfling, Can the mass of the lightest Higgs boson of the minimal supersymmetric model be larger than $m_{Z}$ ?, Phys. Rev. Lett. 66 (1991) 1815 [SPIRES].

[3] J.R. Ellis, G. Ridolfi and F. Zwirner, On radiative corrections to supersymmetric Higgs boson masses and their implications for LEP searches, Phys. Lett. B 262 (1991) 477 [SPIRES].

[4] M.S. Carena, M. Quirós and C.E.M. Wagner, Effective potential methods and the Higgs mass spectrum in the MSSM, Nucl. Phys. B 461 (1996) 407 [hep-ph/9508343] [SPIRES].

[5] M.S. Carena et al., Reconciling the two-loop diagrammatic and effective field theory computations of the mass of the lightest CP-even Higgs boson in the MSSM, Nucl. Phys. B 580 (2000) 29 [hep-ph/0001002] [SPIRES].

[6] K. Inoue, A. Kakuto, H. Komatsu and S. Takeshita, Low-energy parameters and particle masses in a supersymmetric grand unified model, Prog. Theor. Phys. 67 (1982) 1889 [SPIRES].

[7] K. Inoue, A. Kakuto, H. Komatsu and S. Takeshita, Aspects of grand unified models with softly broken supersymmetry, Prog. Theor. Phys. 68 (1982) 927 [Erratum ibid. 70 (1983) 330] [SPIRES].

[8] K. Inoue, A. Kakuto, H. Komatsu and S. Takeshita, Renormalization of supersymmetry breaking parameters revisited, Prog. Theor. Phys. 71 (1984) 413 [SPIRES].

[9] L.E. Ibáñez and G.G. Ross, $\mathrm{SU}(2)_{L} \times U(1)$ symmetry breaking as a radiative effect of supersymmetry breaking in GUTS, Phys. Lett. B 110 (1982) 215 [SPIRES].

[10] L. Álvarez-Gaumé, M. Claudson and M.B. Wise, Low-energy supersymmetry, Nucl. Phys. B 207 (1982) 96 [SPIRES].

[11] L.E. Ibáñez and C. Lopez, $N=1$ supergravity, the weak scale and the low-energy particle spectrum, Nucl. Phys. B 233 (1984) 511 [SPIRES].

[12] L.E. Ibáñez, C. Lopez and C. Muñoz, The low-energy supersymmetric spectrum according to $N=1$ supergravity GUTS, Nucl. Phys. B 256 (1985) 218 [SPIRES]. 
[13] R. Barbieri and G.F. Giudice, Upper bounds on supersymmetric particle masses, Nucl. Phys. B 306 (1988) 63 [SPIRES].

[14] P.H. Chankowski, J.R. Ellis and S. Pokorski, The fine-tuning price of LEP, Phys. Lett. B 423 (1998) 327 [hep-ph/9712234] [SPIRES].

[15] P.H. Chankowski, J.R. Ellis, M. Olechowski and S. Pokorski, Haggling over the fine-tuning price of LEP, Nucl. Phys. B 544 (1999) 39 [hep-ph/9808275] [SPIRES].

[16] G.L. Kane and S.F. King, Naturalness implications of LEP results, Phys. Lett. B 451 (1999) 113 [hep-ph/9810374] [SPIRES].

[17] M. Bastero-Gil, G.L. Kane and S.F. King, Fine-tuning constraints on supergravity models, Phys. Lett. B 474 (2000) 103 [hep-ph/9910506] [SPIRES].

[18] G.L. Kane, J.D. Lykken, B.D. Nelson and L.-T. Wang, Re-examination of electroweak symmetry breaking in supersymmetry and implications for light superpartners, Phys. Lett. B 551 (2003) 146 [hep-ph/0207168] [SPIRES].

[19] A. Brignole, J.A. Casas, J.R. Espinosa and I. Navarro, Low-scale supersymmetry breaking: effective description, electroweak breaking and phenomenology, Nucl. Phys. B 666 (2003) 105 [hep-ph/0301121] [SPIRES].

[20] J.A. Casas, J.R. Espinosa and I. Hidalgo, The MSSM fine tuning problem: a way out, JHEP 01 (2004) 008 [hep-ph/0310137] [SPIRES].

[21] J.A. Casas, J.R. Espinosa and I. Hidalgo, Implications for new physics from fine-tuning arguments. I: application to SUSY and seesaw cases, JHEP 11 (2004) 057 [hep-ph/0410298] [SPIRES].

[22] P. Batra, A. Delgado, D.E. Kaplan and T.M.P. Tait, The Higgs mass bound in gauge extensions of the minimal supersymmetric standard model, JHEP 02 (2004) 043 [hep-ph/0309149] [SPIRES].

[23] K. Agashe and M. Graesser, Improving the fine tuning in models of low energy gauge mediated supersymmetry breaking, Nucl. Phys. B 507 (1997) 3 [hep-ph/9704206] [SPIRES].

[24] R. Harnik, G.D. Kribs, D.T. Larson and H. Murayama, The minimal supersymmetric fat Higgs model, Phys. Rev. D 70 (2004) 015002 [hep-ph/0311349] [SPIRES].

[25] S. Chang, C. Kilic and R. Mahbubani, The new fat Higgs: slimmer and more attractive, Phys. Rev. D 71 (2005) 015003 [hep-ph/0405267] [SPIRES].

[26] A. Delgado and T.M.P. Tait, A fat Higgs with a fat top, JHEP 07 (2005) 023 [hep-ph/0504224] [SPIRES].

[27] T. Kobayashi and H. Terao, Suppressed supersymmetry breaking terms in the Higgs sector, JHEP 07 (2004) 026 [hep-ph/0403298] [SPIRES].

[28] T. Kobayashi, H. Nakano and H. Terao, Induced top Yukawa coupling and suppressed Higgs mass parameters, Phys. Rev. D 71 (2005) 115009 [hep-ph/0502006] [SPIRES].

[29] T. Kobayashi, H. Terao and A. Tsuchiya, Fine-tuning in gauge mediated supersymmetry breaking models and induced top Yukawa coupling, Phys. Rev. D 74 (2006) 015002 [hep-ph/0604091] [SPIRES].

[30] A. Birkedal, Z. Chacko and Y. Nomura, Relaxing the upper bound on the mass of the lightest supersymmetric Higgs boson, Phys. Rev. D 71 (2005) 015006 [hep-ph/0408329] [SPIRES]. 
[31] A. Birkedal, Z. Chacko and M.K. Gaillard, Little supersymmetry and the supersymmetric little hierarchy problem, JHEP 10 (2004) 036 [hep-ph/0404197] [SPIRES].

[32] Z. Chacko, Y. Nomura and D. Tucker-Smith, A minimally fine-tuned supersymmetric standard model, Nucl. Phys. B 725 (2005) 207 [hep-ph/0504095] [SPIRES].

[33] K. Choi, K.S. Jeong, T. Kobayashi and K.-i. Okumura, Little SUSY hierarchy in mixed modulus-anomaly mediation, Phys. Lett. B 633 (2006) 355 [hep-ph/0508029] [SPIRES].

[34] K. Choi, K.S. Jeong, T. Kobayashi and K.-i. Okumura, TeV scale mirage mediation and natural little SUSY hierarchy, Phys. Rev. D 75 (2007) 095012 [hep-ph/0612258] [SPIRES].

[35] R. Kitano and Y. Nomura, A solution to the supersymmetric fine-tuning problem within the MSSM, Phys. Lett. B 631 (2005) 58 [hep-ph/0509039] [SPIRES].

[36] A. Falkowski, S. Pokorski and M. Schmaltz, Twin SUSY, Phys. Rev. D 74 (2006) 035003 [hep-ph/0604066] [SPIRES].

[37] S. Chang, L.J. Hall and N. Weiner, A supersymmetric twin Higgs, Phys. Rev. D 75 (2007) 035009 [hep-ph/0604076] [SPIRES].

[38] G.F. Giudice and R. Rattazzi, Living dangerously with low-energy supersymmetry, Nucl. Phys. B 757 (2006) 19 [hep-ph/0606105] [SPIRES].

[39] R. Dermisek and H.D. Kim, Radiatively generated maximal mixing scenario for the Higgs mass and the least fine tuned minimal supersymmetric standard model, Phys. Rev. Lett. 96 (2006) 211803 [hep-ph/0601036] [SPIRES].

[40] R. Dermisek, H.D. Kim and I.-W. Kim, Mediation of supersymmetry breaking in gauge messenger models, JHEP 10 (2006) 001 [hep-ph/0607169] [SPIRES].

[41] R. Essig and J.-F. Fortin, The minimally tuned minimal supersymmetric standard model, JHEP 04 (2008) 073 [arXiv: 0709.0980] [SPIRES].

[42] I. Gogoladze, M.U. Rehman and Q. Shafi, Amelioration of little hierarchy problem in $\mathrm{SU}(4)_{c} \times \mathrm{SU}(2)_{L} \times \mathrm{SU}(2)_{R}$, Phys. Rev. D $80(2009) 105002$ [arXiv:0907.0728] [SPIRES].

[43] D. Horton and G.G. Ross, Naturalness and focus points with non-universal gaugino masses, arXiv: 0908.0857 [SPIRES].

[44] R. Dermisek, Unusual Higgs or supersymmetry from natural electroweak symmetry breaking, Mod. Phys. Lett. A 24 (2009) 1631 [arXiv:0907.0297] [SPIRES].

[45] P.W. Graham, A. Ismail, S. Rajendran and P. Saraswat, A little solution to the little hierarchy problem: a vector-like generation, arXiv:0910.3020 [SPIRES].

[46] H. Abe, T. Kobayashi and Y. Omura, Relaxed fine-tuning in models with non-universal gaugino masses, Phys. Rev. D 76 (2007) 015002 [hep-ph/0703044] [SPIRES].

[47] K. Choi, A. Falkowski, H.P. Nilles, M. Olechowski and S. Pokorski, Stability of flux compactifications and the pattern of supersymmetry breaking, JHEP 11 (2004) 076 [hep-th/0411066] [SPIRES].

[48] K. Choi, A. Falkowski, H.P. Nilles and M. Olechowski, Soft supersymmetry breaking in KKLT flux compactification, Nucl. Phys. B 718 (2005) 113 [hep-th/0503216] [SPIRES].

[49] K. Choi, K.S. Jeong and K.-i. Okumura, Phenomenology of mixed modulus-anomaly mediation in fluxed string compactifications and brane models, JHEP 09 (2005) 039 [hep-ph/0504037] [SPIRES]. 
[50] M. Endo, M. Yamaguchi and K. Yoshioka, A bottom-up approach to moduli dynamics in heavy gravitino scenario: superpotential, soft terms and sparticle mass spectrum, Phys. Rev. D 72 (2005) 015004 [hep-ph/0504036] [SPIRES].

[51] A. Brignole, L.E. Ibáñez and C. Muñoz, Towards a theory of soft terms for the supersymmetric Standard Model, Nucl. Phys. B 422 (1994) 125 [Erratum ibid. B 436 (1995) 747] [hep-ph/9308271] [SPIRES].

[52] T. Kobayashi, D. Suematsu, K. Yamada and Y. Yamagishi, Nonuniversal soft scalar masses in superstring theories, Phys. Lett. B 348 (1995) 402 [hep-ph/9408322] [SPIRES].

[53] L.E. Ibáñez, C. Muñoz and S. Rigolin, Aspects of type-I string phenomenology, Nucl. Phys. B 553 (1999) 43 [hep-ph/9812397] [SPIRES].

[54] J.R. Ellis, C. Kounnas and D.V. Nanopoulos, No scale supersymmetric GUTS, Nucl. Phys. B 247 (1984) 373 [SPIRES].

[55] J.R. Ellis, K. Enqvist, D.V. Nanopoulos and K. Tamvakis, Gaugino masses and grand unification, Phys. Lett. B 155 (1985) 381 [SPIRES].

[56] M. Drees, Phenomenological consequences of $N=1$ supergravity theories with nonminimal kinetic energy terms for vector superfields, Phys. Lett. B 158 (1985) 409 [SPIRES].

[57] G. Anderson et al., Motivations for and implications of non-universal GUT-scale boundary conditions for soft SUSY-breaking parameters, in the proceedings of 1996DPF/DPB Summer Study on New Directions for High-Energy Physics (Snowmass 96) June 25-July 25, Snowmass, Colorado U.S.A. (1996), hep-ph/9609457 [SPIRES].

[58] K. Huitu, Y. Kawamura, T. Kobayashi and K. Puolamaki, Phenomenological constraints on SUSY SU(5) GUTs with non- universal gaugino masses, Phys. Rev. D 61 (2000) 035001 [hep-ph/9903528] [SPIRES].

[59] S.P. Martin, Non-universal gaugino masses from non-singlet F-terms in non-minimal unified models, Phys. Rev. D 79 (2009) 095019 [arXiv:0903.3568] [SPIRES].

[60] K.J. Bae, R. Dermisek, H.D. Kim and I.-W. Kim, Mixed Bino-Wino-Higgsino dark matter in gauge messenger models, JCAP 08 (2007) 014 [hep-ph/0702041] [SPIRES].

[61] H. Abe, Y.G. Kim, T. Kobayashi and Y. Shimizu, TeV scale partial mirage unification and neutralino dark matter, JHEP 09 (2007) 107 [arXiv: 0706.4349] [SPIRES].

[62] I. Gogoladze, R. Khalid and Q. Shafi, Coannihilation Scenarios and Particle Spectroscopy in $\mathrm{SU}(4)_{c} \times \mathrm{SU}(2)_{L} \times \mathrm{SU}(2)_{R}$, Phys. Rev. D $80(2009) 095016$ [arXiv: 0908. 0731] [SPIRES].

[63] C. Cheung, A.L. Fitzpatrick and D. Shih, (Extra)ordinary gauge mediation, JHEP 07 (2008) 054 [arXiv:0710.3585] [SPIRES].

[64] G.F. Giudice and R. Rattazzi, Theories with gauge-mediated supersymmetry breaking, Phys. Rept. 322 (1999) 419 [hep-ph/9801271] [SPIRES].

[65] P. Meade, N. Seiberg and D. Shih, General gauge mediation, Prog. Theor. Phys. Suppl. 177 (2009) 143 [arXiv:0801.3278] [SPIRES].

[66] L.M. Carpenter, M. Dine, G. Festuccia and J.D. Mason, Implementing general gauge mediation, Phys. Rev. D 79 (2009) 035002 [arXiv:0805.2944] [SPIRES].

[67] H. Ooguri, Y. Ookouchi, C.-S. Park and J. Song, Current correlators for general gauge mediation, Nucl. Phys. B 808 (2009) 121 [arXiv:0806.4733] [SPIRES]. 
[68] J. Distler and D. Robbins, General F-term gauge mediation, arXiv:0807.2006 [SPIRES].

[69] K.A. Intriligator and M. Sudano, Comments on general gauge mediation, JHEP 11 (2008) 008 [arXiv:0807.3942] [SPIRES].

[70] M. Buican, P. Meade, N. Seiberg and D. Shih, Exploring general gauge mediation, JHEP 03 (2009) 016 [arXiv: 0812.3668] [SPIRES].

[71] K. Benakli and M.D. Goodsell, Dirac gauginos in general gauge mediation, Nucl. Phys. B 816 (2009) 185 [arXiv:0811.4409] [SPIRES].

[72] L.M. Carpenter, Surveying the phenomenology of general gauge mediation, arXiv:0812.2051 [SPIRES].

[73] D. Marques, Generalized messenger sector for gauge mediation of supersymmetry breaking and the soft spectrum, JHEP 03 (2009) 038 [arXiv:0901.1326] [SPIRES].

[74] M. Luo and S. Zheng, Gauge extensions of supersymmetric models and hidden valleys, JHEP 04 (2009) 122 [arXiv:0901.2613] [SPIRES].

[75] A. Rajaraman, Y. Shirman, J. Smidt and F. Yu, Parameter space of general gauge mediation, Phys. Lett. B 678 (2009) 367 [arXiv: 0903.0668] [SPIRES].

[76] D. Koschade, M. McGarrie and S. Thomas, Direct mediation and metastable supersymmetry breaking for $\mathrm{SO}(10)$, arXiv:0909.0233 [SPIRES].

[77] M. Buican and Z. Komargodski, Soft terms from broken symmetries, arXiv:0909.4824 [SPIRES].

[78] R. Essig, Implications of the LEP Higgs bounds for the MSSM stop sector, Phys. Rev. D 75 (2007) 095005 [hep-ph/0702104] [SPIRES].

[79] C. Csáki, A. Falkowski, Y. Nomura and T. Volansky, New approach to the $\mu-B \mu$ problem of gauge-mediated supersymmetry breaking, Phys. Rev. Lett. 102 (2009) 111801 [arXiv: 0809.4492] [SPIRES].

[80] S. Abel, M.J. Dolan, J. Jaeckel and V.V. Khoze, Phenomenology of pure general gauge mediation, arXiv:0910.2674 [SPIRES]. 